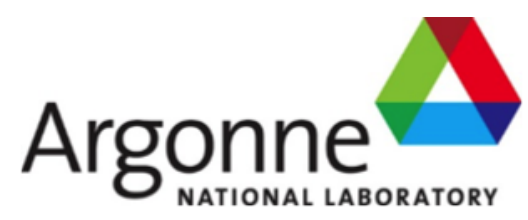

ANL-ART-218

\title{
Microstructural Model for Creep-Fatigue Interaction in Grade 91 Steel
}

Applied Materials Division 
About Argonne National Laboratory

Argonne is a U.S. Department of Energy laboratory managed by UChicago Argonne, LLC under contract DE-AC02-06CH11357. The Laboratory's main facility is outside Chicago, at 9700 South Cass Avenue, Argonne, Illinois 60439. For information about Argonne and its pioneering science and technology programs, see www.anl.gov.

\section{DOCUMENT AVAILABILITY}

Online Access: U.S. Department of Energy (DOE) reports produced after 1991 and a growing number of pre-1991 documents are available free at OSTI.GOV (http://www.osti.gov/), a service of the U.S. Dept. of Energy's Office of Scientific and Technical Information

Reports not in digital format may be purchased by the public from the

National Technical Information Service (NTIS):

U.S. Department of Commerce

National Technical Information Service

5301 Shawnee Rd

Alexandria, VA 22312

www.ntis.gov

Phone: (800) 553-NTIS (6847) or (703) 605-6000

Fax: (703) 605-6900

Email: orders@ntis.gov

Reports not in digital format are available to DOE and DOE contractors from the Office of Scientific and Technical Information (OSTI)

U.S. Department of Energy

Office of Scientific and Technical Information

P.O. Box 62

Oak Ridge, TN 37831-0062

www.osti.gov

Phone: (865) 576-8401

Fax: (865) 576-5728

Email: reports@osti.gov

\section{Disclaimer}

This report was prepared as an account of work sponsored by an agency of the United States Government. Neither the United States Government nor any agency thereof, nor UChicago Argonne, LLC, nor any of their employees or officers, makes any warranty, express or implied, or assumes any legal liability or responsibility for the accuracy, completeness, or usefulness of any information, apparatus, product, or process disclosed, or represents that its use would not infringe privately owned rights. Reference herein to any specific commercial product, process, or service by trade name, trademark, manufacturer, or otherwise, does not necessarily constitute or imply its endorsement, recommendation, or favoring by the United States Government or any agency thereof. The views and opinions of document authors expressed herein do not necessarily state or reflect those of the United States Government or any agency thereof, Argonne National Laboratory, or UChicago Argonne, LLC. 


\section{Microstructural Model for Creep-Fatigue Interaction in Grade 91 Steel}

Applied Materials Division

Argonne National Laboratory

March 2021

Prepared by

M. C. Messner, Argonne National Laboratory

Ajey Venkataraman, Argonne National Laboratory

Andrea Rovinelli, Argonne National Laboratory

T.-L. Sham, Idaho National Laboratory 



\section{Abstract}

This report describes the completion of a crystal plasticity finite element simulation framework for creep-fatigue deformation and damage in Grade 91 steel. In addition to a description of the framework and a set of verification calculations, the report details an initial set of simulations applying the framework to examine low cycle fatigue and creep-fatigue interaction in Grade 91 at $600^{\circ} \mathrm{C}$. Grade 91 is a likely material for future advanced microreactors and creep-fatigue will be a limiting structural damage mechanism, particularly for mobile reactors or load following systems. The framework described here could provide a better understanding of creep-fatigue damage mechanisms and directly examine creep, fatigue, and creep-fatigue interaction for Grade 91 at realistic component service conditions. This in turn could lead to improved design methods and component life estimation approaches, leading to more efficient and economical component designs. 



\section{Table of Contents}

Abstract i i

Table of Contents $\quad$ iii

List of Figures $\quad$ V

List of Tables vii

1 Introduction 1

2 A crystal plasticity framework for transgranular damage 3

2.1 General implementation . . . . . . . . . . . . . . . . . . 4

2.1.1 Basic stress update formula . . . . . . . . . . . . . . . . 4

2.2 Specific models . . . . . . . . . . . . . . . . . . . . . . . . . . . . . . . . . . . . . . .

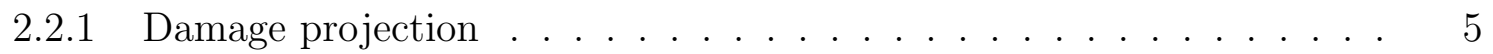

2.2 .2 Damage indicators . . . . . . . . . . . . . . . . 8

2.3 Implementation . . . . . . . . . . . . . . . . . . . . . . . . . . . . . . . . . . . .

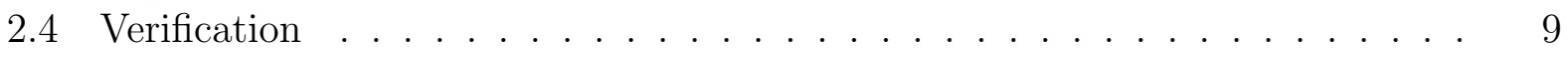

3 Modeling pure fatigue damage 11

3.1 Model setup . . . . . . . . . . . . . . . . . . . . . 11

3.2 Basic fatigue response . . . . . . . . . . . . . . . . . . . 11

3.3 Improving the model . . . . . . . . . . . . . . . . . . . . . 13

4 Initial creep-fatigue simulations $\quad 15$

4.1 Simulate setup . . . . . . . . . . . . . . . . . . . . 15

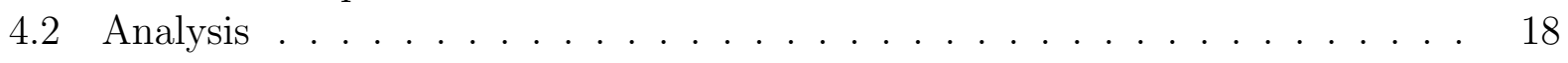

5 Conclusions 25

$\begin{array}{lr}\text { Bibliography } & 29\end{array}$ 



\section{List of Figures}

2.1 Example illustrating the effect of the $c$ and $\beta$ parameters on the sigmoid damage map. . . . . . . . . . . . . . . . . . . . . . . . . . 8

2.2 Basic response of the damage model under monotonic tension. . . . . . . . . 10

2.3 Demonstration that damage changes the effective elastic properties of the Taylor polycrystal. . . . . . . . . . . . . . . . . . 10

2.4 Illustration of the change in the unload/reload slope of the polycrystal caused by the tension/compression switch. . . . . . . . . . . . . 10

3.1 Example of results for a $0.5 \%$ strain range, plotting the cyclic material history as stress-strain hysteresis (a) and a stress-time cyclic softening curve (b). . .

3.2 Results of the fatigue life simulations at $600^{\circ} \mathrm{C}$. The plot shows the nominal ASME fatigue curve for Grade 91 compared to the simulation results. The results match the low cycle part of the curve remarkably accurately, considering that the only calibration was shifting the work-to-failure to match the number of cycles to failure at the highest strain range. . . . . . . . . . . .

4.1 (a) The creep-fatigue loading cycle used in the present work. (b) An example stress-strain curve from a creep-fatigue simulation. An initial hardening response is proceeded by a softening response with increasing cycles. . . . . 16

4.2 The 5 grain meshed microstructure used in the present study. . . . . . . . . 16

4.3 Strain history for the creep-fatigue simulations. The ramps correspond to changes in the applied strains and the flat lines indicate the hold between fatigue cycles. . . . . . . . . . . . . . . . . . .

4.4 Shown above are the stress-strain curves for the creep-fatigue simulations for models with (a) interfacial damage model only, (b) transgranular damage model only and (c) both interfacial and transgranular damage models. . . .

4.5 (a) The mean stress in the loading direction is plotted against time for the three damage models considered. (b) The mean stress plotted against the cycle number. . . . . . . . . . . . . . . . . . . . .

4.6 The maximum stress in the loading direction is plotted against cycle number for the three damage models considered. . . . . . . . . . . . . . . . . . . . . 20

4.7 Comparison of the transgranular model damage metrics for creep-fatigue simulations. . . . . . . . . . . . . . . . . . .

4.8 Difference between the maximum transgranular damage metrics for the combined transgranular + interfacial model and just the interfacial model. . . .

4.9 The evolution of the interfacial damage metric as a function of (a) cycle number and (b) time for the model with only interfacial damage model. . . . . . 22 

Microstructural Model for Creep-Fatigue Interaction in Grade 91 Steel

March 2021

\section{List of Tables}

4.1 The material parameters used in the present simulations. The value of $c$ (the critical work) was greatly decreased to accelerate the development of fatigue damage. . . . . . . . . . . . . . . . . . . 



\section{Introduction}

This report describes the completion of a framework for representing the key creep-fatigue deformation and damage mechanisms in Grade 91 steel. The framework includes several features summarized in past reports:

1. A finite element framework for microstructural full-field crystal plasticity finite element method (CPFEM) simulations of metallic materials [1].

2. An interface-cohesive formulation, implemented as a Discontinuous Galerkin (DG) element interfaces, to model grain boundary creep damage [2]. The particular grain boundary damage model has a long history $[3-5]$ and the current implementation includes a variety of numerical improvements designed to stabilize it for cyclic, creepfatigue loading [6].

3. An improved single-crystal grain bulk constitutive model [6] that can represent kinematic hardening and, in general, capture the cyclic deformation of Grade 91 under creep-fatigue conditions.

The main new development described in this report is the addition of a continuum damage mechanics framework to represent transgranular fatigue damage. When combined with the preexisting work, the complete CPFEM model can represent all the key microstructural deformation and damage mechanisms in Grade 91 contributing to creep-fatigue behavior.

The completion of this framework represents a significant technical advance as, to our knowledge, no one has attempted full-field, microstructural simulations of creep-fatigue behavior in any material. Past work using microstructural methods covers only pure fatigue (c.f. $[7,8]$ ), pure creep (c.f. $[9,10])$, or does not include both grain boundary and transgranular damage (c.f. $[11,12])$.

This report describes the development of the fatigue damage model and simulations assessing the model for pure fatigue loading. In addition to completing the creep-fatigue modeling framework, the transgranular model could be useful in examining the transition from low cycle to high cycle fatigue behavior, associated with a change in slope in a strain-controlled fatigue curve observed experimentally. Finally, the report summarizes a preliminary series of full-field CPFEM simulations aimed at examining creep-fatigue interaction mechanisms in Grade 91 at $600^{\circ} \mathrm{C}$.

Creep-fatigue damage has historically been a significant design limitation for nuclear components constructed of Grade 91 and other advanced materials [13] and we expect that it will similarly constrain future reactor designs, including microreactors. In fact, cyclic load and creep-fatigue may be an even more significant concern for high temperature microreactors, particularly those designed for portability or load following, when compared to larger reactors. The reason is that creep-fatigue damage is proportional to the number of load cycles, often associated with thermal transients, and so a reactor with a greater number of expected transients will likely accumulate more creep-fatigue damage compared to a larger reactor operating in steady conditions. Creep-fatigue damage will also likely be the limiting failure mode for low pressure, high temperature reactor designs, as creep damage in the steady state due to pressure for these reactors is often negligible. 
The key macroscale structural material phenomenon of interest to reactor designers for loading conditions of this sort, combining thermal cycling with holds at quasi-steady conditions, is creep-fatigue interaction. Creep-fatigue interaction in the engineering sense is the reduction in cyclic life associated with the combination of cycling plus holds at constant conditions versus just the cycling itself. For example, experimentally the cyclic life, expressed as the number of cycles to failure, for an experiment on a material combining a strain controlled loading with holds at constant strain on one or both ends of the cyclic will be shorter than if the same material was loaded under the same strain-controlled fatigue loading without the holds.

Most past work associates creep-fatigue interaction with the combination of fatigue damage due to cycling plus creep damage accumulated during the hold periods. Most or all high temperature structural materials, and all those qualified for use in high temperature components in Section III, Division 5 of the ASME Boiler and Pressure Vessel Code covering high temperature reactors, have a significant creep-fatigue interaction that must be accounted for in a component design [14].

The modeling framework developed here can serve two functions in advancing our understanding of and modeling capabilities for creep-fatigue interaction, leading to more accurate design methods and safer, more effective and economical component designs:

1. Despite a large body of experimental work, the exact microstructural mechanism causing creep-fatigue interaction is not fully described. Damage under pure fatigue conditions generally manifests as transgranular cracking [15]. Damage under pure creep conditions is generally intergranular grain boundary cavitation [16]. Previous work presents a variety of potential interaction mechanisms, including wedge cracking induced by grain boundary cavitation and sliding assisting fatigue crack growth [17] or a variety of mechanisms depending on the particular type of loading [18]. Some past work suggests that the free surface plays a key role, with fatigue cracks propagating from the surface interacting with creep cavitation in the bulk. A full-field microstructural model could explain the interaction mechanism, leading to better, more predictive models.

2. Subject to limitations caused by the computational cost of the model, CPFEM simulations could be used to directly quantify the effect of creep-fatigue interaction in Grade 91, providing synthetic experimental data that could be used to supplement actual experimental results.

Additionally, the modeling framework can provide similar information for pure fatigue loading at high temperatures. An initial demonstration in this report suggests the model is quite accurate in predicting fatigue curves with very limited direct test data, at least for the low cycle regime.

Chapter 2 describes the development, implementation, and verification of the transgranular damage model. Chapter 3 then describes a preliminary study of pure fatigue deformation in Grade 91. Chapter 4 describes a series of full-field simulations of creep-fatigue in Grade 91, completing the FY21 project work, and Chapter 5 summarizes the key results of this study and discusses potential future work using the damage modeling framework. 


\section{A crystal plasticity framework for transgranular damage}

As described in Chapter 1, the main missing ingredient of a creep-fatigue simulation framework in the CPFEM model is a means to represent transgranular fatigue damage. This chapter describes the development and implementation of a continuum damage mechanics based [19] fatigue damage model in the NEML single crystal constitutive model framework.

Previous literature efforts modeling fatigue in the context of crystal plasticity, typically, in full field CPFEM or CPFFT simulations, fall into three categories:

1. Full field simulations examining the mechanical forces (stress, strain, or some fatigue indicating parameter or FIP) leading to fatigue, but not modeling the effect of fatigue damage on the material's mechanical behavior. These studies are by far the most numerous (c.f. [11, 12, 20-22]) and sometimes link to microscale resolution experiments (c.f. $[23])$

2. Simulations that examine short crack propagation in the microstructure typically either through discrete crack remeshing or a cohesive zone approach [24, 25].

3. A continuum damage mechanics approach which tracks an internal variable (or set of internal variables) in the single-crystal constitutive model representing the softening effect of the development of small fatigue cracks (c.f. [8, 26]). These models can represent discrete cracks but only in the sense of a smeared crack [27].

Some previous models span more than one category. For example Proudhon et al. [7] tracks a FIP and does not feed back the development of fatigue damage as softening to the constitutive response (approach \#1), but then discretely meshes and remeshes a crack when the FIP hits a critical value (approach \#2).

All three approaches have advantages and disadvantages, but we elected to implement approach \#3: a continuum damage mechanics model for transgranular fatigue damage. This approach has several key advantages:

1. It has a direct connection with classical FIP development, so suitable microstructural FIPs can be directly integrated into the damage framework.

2. Damage mechanics can account for the softening effect of very small, subgrain scale cracks.

3. At least approximately, the damage model can propagate fatigue damage in the form of small cracks.

4. It does not presuppose damage nucleation sites or a set of potential crack growth paths.

There are numerous crystal plasticity models incorporating damage mechanics concepts (c.f. [28-30]), though comparatively few aimed at simulating fatigue and none, to our knowledge, examining creep-fatigue interaction. The reason for this comparative lack of interest may be the downsides of the continuum damage approach:

1. Continuum damage models, at least those not regularized by including higher-order derivative information, have a well-known mesh-size dependence [31]. 
2. Once damage reaches the scale of a small crack the approach is less accurate than a fracture mechanics approach, for example using cohesive zone models like our model for grain boundary creep damage [4].

3. The approach adds significantly to the computation cost of the model, versus a deformation model. This is significant as, as described in Chapter 1, running a CPFEM simulation through a realistic loading history is already quite computationally expensive.

The following section describes the development of a suitable continuum damage model for transgranular damage in a general context. In fact, the implement in NEML is modular so that we could explore a range of FIPs and damage projections. The section after next then describes the particular models implemented for representing fatigue damage in Grade 91.

\subsection{General implementation}

\subsubsection{Basic stress update formula}

Continuum damage mechanics models start from the constitutive hypothesis:

$$
\boldsymbol{\sigma}=\mathfrak{D}\left(\boldsymbol{\sigma}^{\prime}, \mathbf{d}\right)
$$

where $\boldsymbol{\sigma}$ is the damaged Cauchy stress, $\boldsymbol{\sigma}^{\prime}$ is the undamaged stress response of the material, $\mathbf{d}$ is some set of internal variables describing the evolution of damage, and $\mathfrak{D}$ is the damage model, mapping the undamaged response to the damaged response as a function of the damage variables. Fatigue damage in single crystals is likely to be anisotropic, even on a continuum scale. For example, certain more active slip planes (persistent slip bands) are likely to develop more damage than other, less active slip planes. To accommodate this anisotropy the NEML damage framework uses the fundamental equation:

$$
\boldsymbol{\sigma}=\boldsymbol{P}(\boldsymbol{h}): \boldsymbol{\sigma}^{\prime}
$$

where $\mathbf{P}$ is a fourth order tensor acting as a damage projection mapping the undamaged Cauchy stress to the damaged Cauchy stress as a function of the damage variables, a subset of the general set of model internal variables, $\mathbf{h}$.

The stress-evolution equation implied by this fundamental assumption is

$$
\begin{gathered}
\dot{\boldsymbol{\sigma}}=\dot{\boldsymbol{P}}: \boldsymbol{\sigma}^{\prime}+\boldsymbol{P}: \dot{\boldsymbol{\sigma}}^{\prime} \\
\dot{\boldsymbol{\sigma}}=\dot{\boldsymbol{P}}: \boldsymbol{P}^{-1}: \boldsymbol{\sigma}+\boldsymbol{P}: \dot{\boldsymbol{\sigma}}^{\prime} .
\end{gathered}
$$

Therefore

$$
\dot{\boldsymbol{\sigma}}=\frac{\partial \boldsymbol{P}}{\partial \boldsymbol{h}}: \dot{\boldsymbol{h}}: \boldsymbol{P}^{-1}: \boldsymbol{\sigma}+\boldsymbol{P}: \dot{\boldsymbol{\sigma}}^{\prime}
$$

Here we make one more significant constitutive assumption: that the subset of the internal variables affecting the damage projection (the damage variables) evolve slowly with time so that

$$
\dot{h} \approx 0 \text {. }
$$


This assumption is reasonable for processes like creep and fatigue, which evolve very slowly with time, and greatly simplifies the damaged stress evolution equation:

$$
\dot{\boldsymbol{\sigma}}=\boldsymbol{P}: \dot{\boldsymbol{\sigma}}^{\prime}
$$

Equation 2.7 is the key damage equation implemented in NEML. The framework takes as input the undamaged stress evolution rate equation from crystal plasticity forms already implemented in NEML and returns the modified, damaged stress evolution rate. A complete model supplements this basic stress update formula with

1. The form of the damage projection

2. A set of internal variables representing the driving force for degradation and failure in the material (the damage variables).

However, fatigue damage modeling can take advantage of a more general framework allowing the projection to vary with the stress, as described below, and so the implementation retains the general form in 2.7 .

NEML implements the standard Asaro [32] kinematics for crystal plasticity and so the modified (damaged) evolution equation for the Cauchy stress becomes:

$$
\dot{\sigma}_{i j}=P_{i j k l} C_{k l m n}\left(D_{m n}-D_{m n}^{p}\right)-\sigma_{i k} \Omega_{k j}^{\star}+\Omega_{i k}^{\star} \sigma_{k j} .
$$

All the subsequent results use this stress evolution formulation.

\subsection{Specific models}

\subsubsection{Damage projection}

\subsubsection{Basic form}

Most microstructural FIPs base damage on slip planes [33]. As such, the damage projection operator implemented here for modeling Grade 91 decomposes damage across multiple planes. Within each single plane the projection allows independent control over the reduced normal and shear stiffness.

Consider the base projection operators:

$$
\begin{gathered}
N_{i j k l}^{(i)}=n_{i}^{(i)} n_{j}^{(i)} n_{k}^{(i)} n_{l}^{(i)} \\
S_{i j k l}^{(i)}=\left(\delta_{i k}-n_{i}^{(i)} n_{k}^{(i)}\right) n_{j}^{(i)} n_{l}^{(i)}
\end{gathered}
$$

where $n_{i}^{(i)}$ is the normal vector for plane $i$.

The damage model uses these projections in conjunction with a mapped damage variable transformation $T$ (the classic "damage" in continuum damage mechanics) where

$$
T=0
$$

indicates an undamaged material plane and

$$
T=1
$$


indicates complete failure (no stiffness).

Putting these concepts together, consider normal damage given by

$$
P_{i j k l}^{n o r m a l,(i)}=\delta_{i k} \delta_{j l}-N^{(i)} N_{i j k l}^{(i)}
$$

where $N^{(i)}$ is the mapped scalar normal damage. When $N^{(i)}=1$ then the resolved stress in the normal direction of the normal traction of the projected stress is

$$
n_{i}^{(i)} P_{i j k l}^{n o r m a l,(i)} \sigma_{k l} n_{j}^{(i)}=n_{i}^{(i)}\left(\delta_{i k} \delta_{j l}-n_{i}^{(i)} n_{j}^{(i)} n_{k}^{(i)} n_{l}^{(i)}\right) \sigma_{k l} n_{j}^{(i)}=n_{i}^{(i)} \sigma_{i j} n_{j}^{(i)}-n_{i}^{(i)} \sigma_{i j} n_{j}^{(i)}=0
$$

So that as the normal mapped damage goes to 1 the normal stress goes to zero.

Similarly, for shear damage given by

$$
P_{i j k l}^{s h e a r,(i)}=\delta_{i k} \delta_{j l}-S^{(i)} S_{i j k l}^{(i)}
$$

where $S^{(i)}$ is the mapped shear damage, when $S^{(i)}=1$ then the normal traction in any direction $s_{i}$ orthogonal to $n_{i}$ becomes

$$
s_{i}^{(i)} P_{i j k l}^{\text {shear },(i)} \sigma_{k l} n_{j}^{(i)}=s_{i}^{(i)}\left(\delta_{i k} \delta_{j l}-\left(\delta_{i k}-n_{i}^{(i)} n_{k}^{(i)}\right) n_{j}^{(i)} n_{l}^{(i)}\right) \sigma_{k l} n_{j}^{(i)}=s_{i}^{(i)} \sigma_{i j} n_{j}^{(i)}-s_{i}^{(i)} \sigma_{i j} n_{j}^{(i)}=0
$$

Again, this means that as the mapped shear damage goes to 1 the shear stress on the plane goes to zero.

These individual, planar projections are then the natural basis for a complete planar damage model. We elect to use a multiplicative decomposition to combine damage across multiple planes:

$$
P_{i j k l}=\prod_{i=1}^{n_{\text {planes }}} \delta_{i j} \delta_{k l}-N\left(d^{(i)}, \sigma_{\perp}^{(i)}\right) N_{i j k l}^{(i)}-S\left(d^{(i)}, \sigma_{\perp}^{(i)}\right) S_{i j k l}^{(i)}
$$

A multiplicative projection, as opposed to a additive decomposition, ensures that the resolved stresses in the appropriate directions of damaged the damaged Cauchy stress are zero when using large deformation kinematics. However, it comes with the added expense and complexity of a difficult Jacobian when implemented in an implicit time integration framework. While the formulation is general, in the context of the Grade 91 crystal plasticity modeling the sum proceeds over each slip plane. For the Grade 91 model used here, that implies a sum over the six $\{110\}$ planes for the $<111>$ directions.

\subsubsection{Damage map functions}

In the damage framework, a damage map (or damage transformation) is a function that maps:

$$
\mathbb{R}^{+} \rightarrow[0,1]
$$

i.e. that maps a positive damage indicator (for example a FIP) to the range $[0,1]$ where 0 indicates no damage and 1 indicates complete failure. These maps serve two purposes: 
1. They normalize damage indicators with arbitrary scales to the standard range required for the projections.

2. They can add additional physics into the base model, for example by changing the effect of damage depending on the sign of the stress resolved on the plane.

While the damage framework can implement general damage maps of the form:

$$
T=T(\boldsymbol{d}, \boldsymbol{\sigma})
$$

where $\boldsymbol{d}$ is a set of internal variables and $\boldsymbol{\sigma}$ is the full stress tensor the models here use the simplified form:

$$
T=T\left(d^{(i)}, \sigma_{\perp}^{(i)}\right)
$$

where $d^{(i)}$ is a scalar damage indicator variable (one for each slip plane) and $\sigma_{\perp}^{(i)}$ is the normal stress,

$$
\sigma_{\perp}^{(i)}=\sigma_{i j} n_{i}^{(i)} n_{j}^{(i)}
$$

The Grade 91 examples here uses three damage map functions.

Sigmoid transform The purpose of this damage map is simply to transform a positive damage indicating parameter to the correct $[0,1]$ range. It takes the form of a sigmoid function:

$$
T\left(d^{(i)}, \sigma_{\perp}^{(i)}\right)= \begin{cases}\frac{1}{1+\left(\frac{d^{(i)}}{c-d^{(i)}}\right)^{-\beta}} & d^{(i)}<c \\ 1 & d^{(i)} \geq c\end{cases}
$$

Figure 2.1 plots the damage map for different values of $c$ and $\beta$. $c$ controls the critical value of the damage parameter, i.e. the location of the ramp in the sigmoid function. $\beta$ controls the slope of the ramp, higher values of $\beta$ produce sharper ramps which in turn provide a quicker onset of the effect of damage in the model.

Damage cutoff This is a meta map in that it takes the results of another damage map and modifies it. It provides a simple cutoff:

$$
T\left(d^{(i)}, \sigma_{\perp}^{(i)}\right)= \begin{cases}\tilde{T}\left(d^{(i)}, \sigma_{\perp}^{(i)}\right) & d^{(i)}<T_{\text {crit }} \\ T_{\text {crit }} & d^{(i)} \geq T_{\text {crit }}\end{cases}
$$

where $T_{\text {crit }}$ is a cutoff on the maximum permissible damage and $\tilde{T}\left(d^{(i)}, \sigma_{\perp}^{(i)}\right)$ is the base, underlying damage map. Some of the calculations described here apply this cutoff function to improve the stability of the model by preventing excessive softening.

Tension/compression switch This is another meta map. This function turns off the effect of damage when the normal stress on the plane is compressive, approximating contact across the faces of a crack. The function is:

$$
T\left(d^{(i)}, \sigma_{\perp}^{(i)}\right)= \begin{cases}\tilde{T}\left(d^{(i)}, \sigma_{\perp}^{(i)}\right) & \sigma_{\perp}^{(i)} \geq 0 \\ 0 & \sigma_{\perp}^{(i)}<0\end{cases}
$$




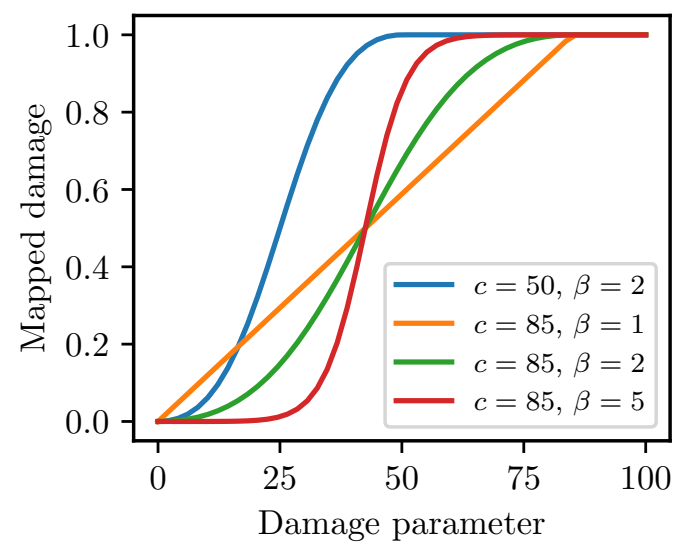

Figure 2.1: Example illustrating the effect of the $c$ and $\beta$ parameters on the sigmoid damage map.

\subsubsection{Damage indicators}

The only requirements for a damage indicator in this framework is that it be an internal variable defined by an evolution equation on each plane in the damage formulation (each slip plane for crystal plasticity models), that it be monotonic, and that it start at a value of 0 . The damage maps then inject the indicator into the appropriate range for the projection operator.

Three fatigue indicating parameters (FIPs), with variants, dominate the literature for microstructural models of fatigue damage [33]:

1. The integrated slip: $\dot{d}^{(i)}=\sum_{j \in S_{i}}\left|\dot{\gamma}_{j}\right|$

2. The integrated work: $\dot{d}^{(i)}=\sum_{j \in S_{i}} \tau_{j}\left|\dot{\gamma}_{j}\right|$

3. The Fatemi-Socie parameter [34]: $\dot{d}^{(i)}=\sum_{j \in S_{i}}\left|\dot{\gamma}_{j}\right|\left(1+K \frac{\sigma_{\perp}^{(i)}}{\sigma_{y}}\right)$ or

$$
\dot{d}^{(i)}=\sum_{j \in S_{i}} \tau_{j}\left|\dot{\gamma}_{j}\right|\left(1+K \frac{\sigma_{\perp}^{(i)}}{\sigma_{y}}\right) .
$$

In these expressions $\dot{\gamma}_{j}$ is the slip rate and $\tau_{j}$ the resolved shear along slip system $j, \sigma_{y}$ is the material yield stress, $K$ is a material-specific constant, and the set $S_{i}$ consists of all slip systems sharing slip plane $i$.

The variants of the Fatemi-Socie parameter are the most general models, but they require an additional material parameter $K$. As such, the Grade 91 examples here use the integrated work as the damage (fatigue) indicating parameter.

\subsection{Implementation}

We implemented this damage model framework into NEML (https://github.com/Arg onne-National-Laboratory/neml) where it sits on top of the existing crystal plasticity 
(deformation) material model library [6]. The implementation is modular, making it easy to implement various damage projections, damage maps, and damage indicators. NEML integrates the single crystal constitutive equations with an implicit scheme, meaning the implementation includes both the rate forms and the Jacobians of the damage models.

In total this required about 10,000 additional lines of code. NEML verifies the mathematical implementation of the damage framework through 38 unit tests which check both the rate form and the derivatives of each individual module.

\subsection{Verification}

The unit tests ensure that the damage model mathematical implementation is correct and that crystal plasticity models including damage will converge quadratically when integrated with NEML's default backward Euler integration and the Newton-Raphson method. The fatigue and creep-fatigue examples in Chapters 3 and 4 demonstrate that the framework can represent the key mechanisms leading to fatigue and creep-fatigue failure in Grade 91 and that the framework can reproduce macroscale experimental data. This section checks that the damage framework implements the intent behind the model mathematics with several simple checks.

The simulations here use a Taylor homogenization of 100 random orientations. The base single crystal deformation model is a simple FCC material with Voce isotropic hardening. The damage transformation for the shear directions is the sigmoid function with $c=40$ and $\beta=3$, while the normal directions stack that same sigmoid function with the tension/compression switch, so that planes under compression approximate fatigue crack face contact.

The damage models developed here target transgranular fatigue but the crystal damage model described above will eventually fail with enough monotonic strain. Figure 2.2 plots a stress/strain curve describing the model response, for example the behavior of the material in a standard tension test. This example verifies that the model captures the basic expected material response: an elastic region, a region of inelasetic deformation with minimal damage, the onset and development of damage, and then, eventually, rapid failure manifested as a loss of load carrying resistance.

Figure 2.3 repeats the same loading conditions but now interrupts the monotonic tension loading before complete failure, reversing the load and unloading the model. This example demonstrates by comparing the load and unload elastic slopes that damage degrades the materials effective elastic modulus. The degradation of the material elastic modulus correctly represents the effects of damage processes in actual materials.

Finally, Figure 2.4 continues the reverse loading until reyield in the compressive direction. This example verifies the damage model can accommodate cyclic plasticity, necessary to represent fatigue and creep-fatigue loading conditions. This test also demonstrates the effect of the tension/compression switch in the damage map. The initial unloading slope is less steep than the eventual compression load elastic slope. The beginning branch of this elastic response samples the degraded (damaged) elastic properties but as the load on the individual crystals becomes predominately compress this switches to sample the undegraded (crack face contact) elastic properties. 
Microstructural Model for Creep-Fatigue Interaction in Grade 91 Steel

March 2021

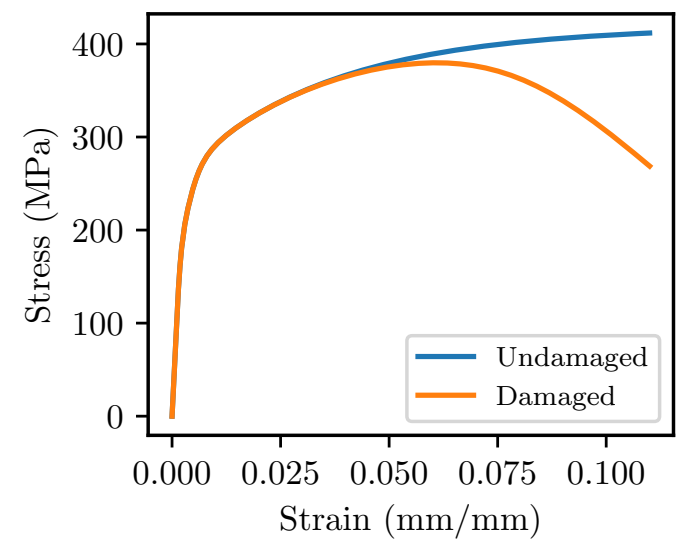

Figure 2.2: Basic response of the damage model under monotonic tension.

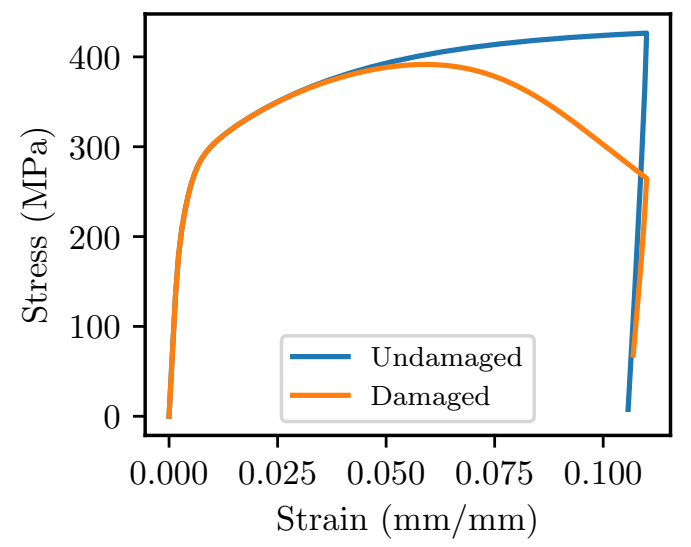

Figure 2.3: Demonstration that damage changes the effective elastic properties of the Taylor polycrystal.

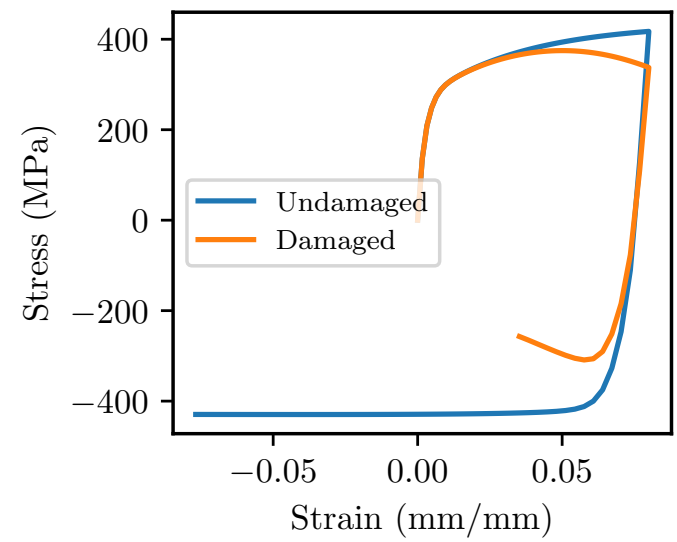

Figure 2.4: Illustration of the change in the unload/reload slope of the polycrystal caused by the tension/compression switch. 


\section{Modeling pure fatigue damage}

While the main objective for the transgranular damage model described in Chapter 2 is to contribute to full-field CPFEM models of creep-fatigue interaction in Grade 91, it is worthwhile to first examine pure fatigue damage at high temperatures. This chapter describes a preliminary model for fatigue at $600^{\circ} \mathrm{C}$ in Grade 91 , using the deformation model established for Grade 91 in past work [6] supplemented with the transgranular fatigue continuum damage model described previously.

The advantage of pure fatigue simulations is that grain boundary damage is a not a significant damage mechanism, meaning reduced cost homogenized models could potentially replace full-field CPFEM simulations. This chapter tests the feasibility of this approach, describes the basic deformation/damage response of the material, and assesses the behavior of homogenized models under pure fatigue loading.

\subsection{Model setup}

The basic model is a Taylor homogenized polycrystal with 100 grains sampled from a uniform texture. The single crystal deformation model matches our past work on Grade 91 [6] for $600^{\circ} \mathrm{C}$. The damage model is as described in the previous chapter, using the sigmoid damage map for both the shear and normal directions with $\beta=2.0$ and a cutoff of $d_{c u t}=0.4$.

To calibrate the $c$ parameter, which is kept the same for both the shear and normal directions, we found the value of $c$ so that the homogenized simulation failed in the same number of cycles as the nominal fatigue curve for Grade 91 in the 2021 edition of the ASME Boiler \& Pressure Vessel Code [14] with a total strain range of $0.1 \mathrm{~mm} / \mathrm{mm}$, a load ratio of $R=-1$, and a strain rate of $10^{-3} \mathrm{~mm} / \mathrm{mm} / \mathrm{s}$. These conditions match common experimental practice when generating data for strain-base high temperature fatigue curves. The calibrated value is $c=4600 \mathrm{MPa}$. We define failure for all these simulations as when the maximum stress over the past cycle is less than $20 \%$ of the highest maximum stress observed for any previous cycle. This definition matches standard experimental practice for strain-controlled fatigue tests.

Figure 3.1 plots the model response as both stress/strain hysteresis loops and as a plot of the maximum/minimum cycle stress as a function of cycle count (a cyclic flow curve). These plots demonstrate that the model response is reasonable for actual Grade 91 material under these loading conditions, demonstrating nearly immediate cyclic softening. In actuality, the onset of softening may occur too quickly, likely because of the lack of constraint from neighboring grains in the Taylor simulation.

\subsection{Basic fatigue response}

After calibrating the model as described in the previous section we repeated the fatigue loading for smaller values of strain range, keeping the $R$ ratio and strain rate fixed. Figure 3.2 compares the results of this prediction of the Grade 91 fatigue curve to the nominal fatigue curve used in the ASME Code. The fit is remarkably accurate in the low cycle region, particularly as there are no parameters to control the slope of the fatigue curve as a function of cycles. 


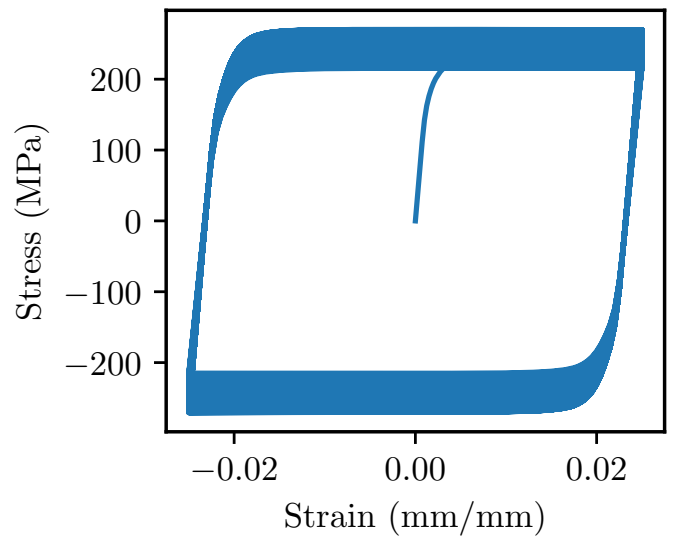

(a) Stress-strain response

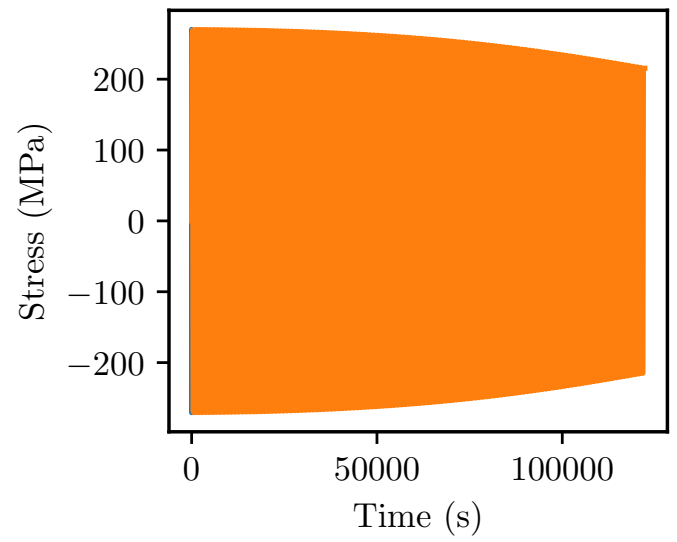

(b) Stress-time response

Figure 3.1: Example of results for a $0.5 \%$ strain range, plotting the cyclic material history as stress-strain hysteresis (a) and a stress-time cyclic softening curve (b).

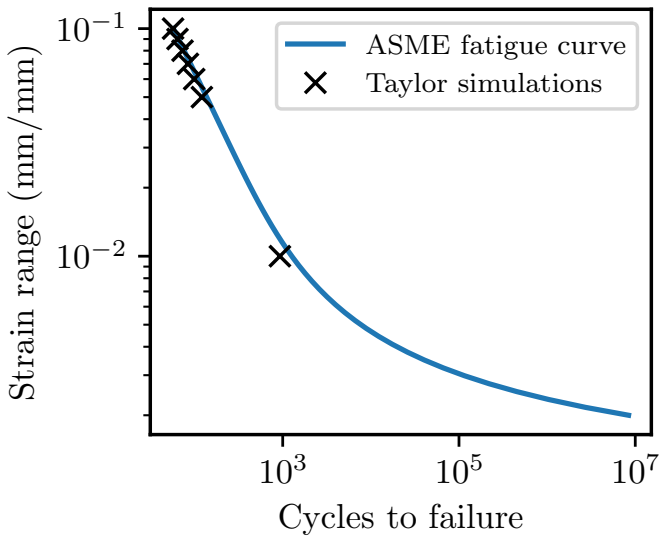

Figure 3.2: Results of the fatigue life simulations at $600^{\circ} \mathrm{C}$. The plot shows the nominal ASME fatigue curve for Grade 91 compared to the simulation results. The results match the low cycle part of the curve remarkably accurately, considering that the only calibration was shifting the work-to-failure to match the number of cycles to failure at the highest strain range. 


\subsection{Improving the model}

There are several ways we could improve this model. The Taylor approximation forces all grains in the polycrystal to deform at the same rate. This means, for strain controlled loading, that when one grain develops damage and eventually fails there is no load redistribution to the remaining, less-damaged grains. This likely slows the development of fatigue damage, especially near the end of the simulation. Improved homogenization techniques, like the selfconsistent viscoplastic approach [35], provide more realistic load redistribution for straincontrolled loading and could improve the accuracy of the model without significantly adding to the computational cost.

A full field simulation, like the creep-fatigue simulations described in the next chapter, would provide additional realism, albeit at much greater computational cost. For example, the homogenized simulations cannot account for strain localization to persistent slip bands, the constraint provided by neighboring grains, or the effects of grain boundaries. Full field simulations of fatigue loading would include these effects. However, the computational cost of running simulations to realistic numbers of fatigue cycles is likely too high, even for modern HPC.

One of the interesting behaviors that we could examine with homogenized pure fatigue crystal plasticity simulations is the transition from low to high cycle fatigue As illustrated in Figure 3.2, a "knee" occurs in experimental fatigue curves, associated with the transition between high and low cycle behavior. Homogenized crystal plasticity models could produce results for the number of fatigue cycles required to sample this behavior.

Computational cost will be a challenge for examining the high cycle transition, as it takes quite awhile to run even the homogenized model through more than 10,000 load cycles. Time acceleration (i.e. cycle extrapolation) is an option to reduce the cost of the simulations [36]. In effect this extrapolates the model results as a function of cycle count, jumping ahead some predetermined number of cycles by extrapolating the stress and internal variables in the model as a function of cycles. The best time acceleration algorithms include error estimates obtained through direct integration of cycles interspersed with extrapolation in order to maintain integration accuracy. 



\section{Initial creep-fatigue simulations}

\subsection{Simulate setup}

Creep-fatigue experiments are performed at a fixed temperature, under strain controlled loading conditions. Experimental observations of the interaction between the two mechanisms of creep and fatigue have suggested that a strain-controlled cyclic test will have a shorter number of cycles to failure if holds at constant strain are included in comparison to a corresponding test at the same conditions without a hold. However, there is no general agreement in the literature on the mechanism causing creep-fatigue interaction. A typical creep-fatigue cycle is characterized by a maximum strain, $\varepsilon_{\max }$, a minimum strain, $\varepsilon_{\min }$, a hold time at maximum strain, $H_{\varepsilon_{\max }}$ and a hold time at minimum strain, $H_{\varepsilon_{\min }}$. Here, the creep-fatigue cycle is characterized by the following parameters - a maximum strain of $\varepsilon_{\max }=1 \%$, a minimum strain of $\varepsilon_{\min }=-1 \%$, a hold time at the maximum strain, $H_{\varepsilon_{\max }}=10 \mathrm{~h}$, and a hold time at the minimum strain, $H_{\varepsilon_{\min }}=10 \mathrm{~h}$. The strain rate used during the transients is $2 \times 10^{-4} s^{-1}$, which is a standard strain rate for creep-fatigue simulations. The creep-fatigue loading cycle used in the present work is shown in Figure 4.1 (a). The corresponding stress-strain curve is shown in Figure 4.1 (b). These simulations are performed on a 5-grain microstructure with $\sim 1600$ elements, the meshed microstructure is shown in Figure 4.2.

The present work compares the results from creep-fatigue simulations for three types of models:

(i) Intergranular damage model only — in this model, damage is described through grain boundary cavity nucleation and growth. There is also accommodation of grain boundary sliding. The grain interiors are modeled using a standard crystal plasticity framework.

(ii) Transgranular damage model only — as described in Chapter 2, damage is modeled by means of degradation of the stress tensor for grain interiors.

(iii) Intergranular and transgranular damage model — here, damage is accounted for by both the above models.

The damage metrics for the transgranular damage model, as described in Chapter 2, is the integrated work:

$$
\dot{d}^{(i)}=\sum_{j \in S_{i}} \tau_{j}\left|\dot{\gamma}_{j}\right|
$$

The damage metric for the interfacial damage model is described in the form of $D$ in the grain boundary cavitation model as the ratio between the cavity half radius, $a$, and cavity half spacing, $b$ :

$$
D=\frac{a}{b}
$$

The material parameters used for the crystal plasticity are shown in Table 4.1. The parameters were described in an earlier report using this model [6]. 


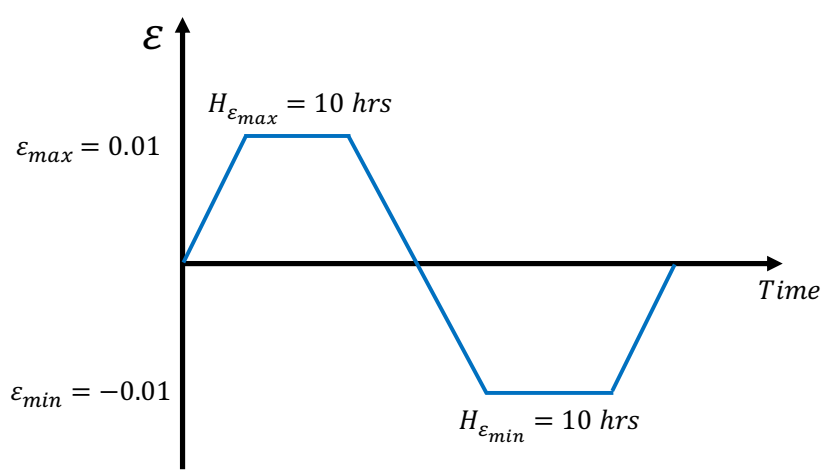

(a)

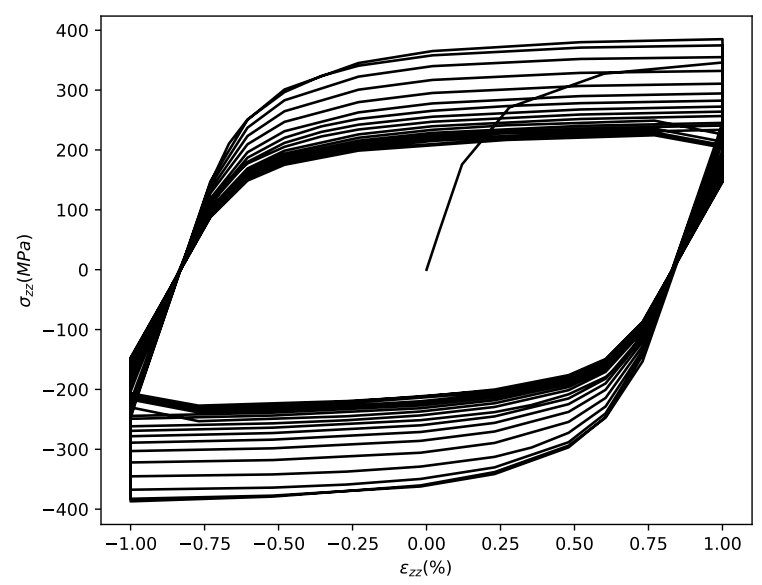

(b)

Figure 4.1: (a) The creep-fatigue loading cycle used in the present work. (b) An example stress-strain curve from a creep-fatigue simulation. An initial hardening response is proceeded by a softening response with increasing cycles.

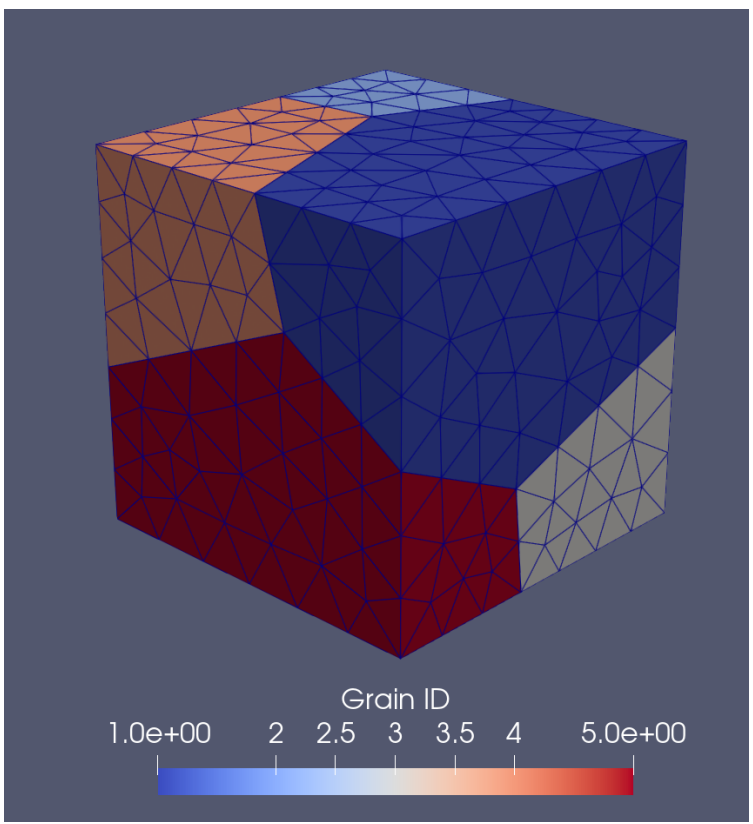

Figure 4.2: The 5 grain meshed microstructure used in the present study. 
Table 4.1: The material parameters used in the present simulations. The value of $c$ (the critical work) was greatly decreased to accelerate the development of fatigue damage.

\begin{tabular}{cccc}
\hline \hline Symbol & Description & Value & Units \\
\hline$E$ & Young's modulus & 150,000 & $\mathrm{MPa}$ \\
$\nu$ & Poisson's ration & 0.285 & unitless \\
$\tau_{\text {sat }}$ & Isotropic saturation & 12 & $\mathrm{MPa}$ \\
$\tau_{0}$ & Isotropic initial value & 40 & $\mathrm{MPa}$ \\
$\dot{\gamma}_{0}$ & Reference slip rate & $9.55 \times 10^{-8}$ & unitless \\
$n$ & Flow exponent & 12 & unitless \\
$\beta$ & Slope of the sigmoid ramp & 2 & unitless \\
$c_{\text {accel }}$ & Critical value of damage parameter & 100 & unitless \\
\hline
\end{tabular}




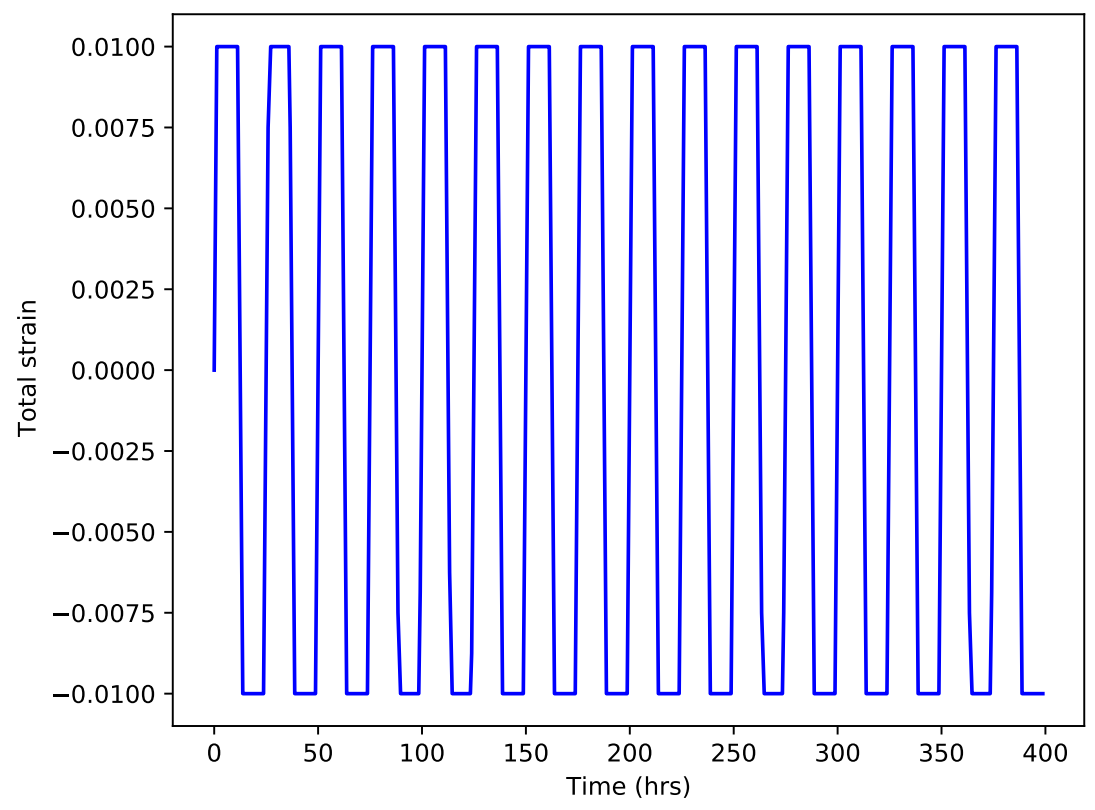

Figure 4.3: Strain history for the creep-fatigue simulations. The ramps correspond to changes in the applied strains and the flat lines indicate the hold between fatigue cycles.

\subsection{Analysis}

Creep-fatigue simulations are performed for the three cases mentioned above and the results are analyzed for differences in accommodation of stress, strain and damage metrics. The strain history for these simulations is shown in Figure 4.3, corresponding to the schematic shown in Figure 4.1 (a). The stress-strain curves for the three damage models throughout the 14 creep-fatigue cycles performed are shown in Figure 4.4. The interfacial damage model reaches a saturation stress within a couple of cycles, after an initial cyclic hardening. Whereas for the other two models, the saturation stress is reached after many more cycles. The homogenized mean stress history for the three cases is compared in Figure 4.5 (a). While only initial hardening is observed for the interfacial damage model, significant softening is observed with increasing fatigue cycles. Also compared in Figure 4.5 (b) is the homogenized stress values as a function of fatigue cycle count. The main difference observed here is that the simulations with only the interfacial damage experiences a compressive average stress and this is attributed to the activation of the grain boundary cavitation damage only during tensile portion of the fatigue cycles. Also shown in Figure 4.6 is the maximum stress per cycle for the three models. The softening response of the two models with transgranular damage is evident here. 


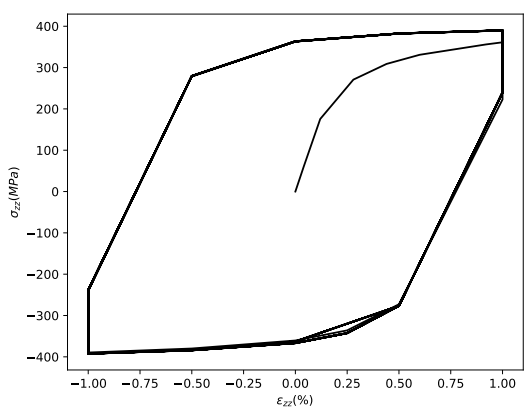

(a)

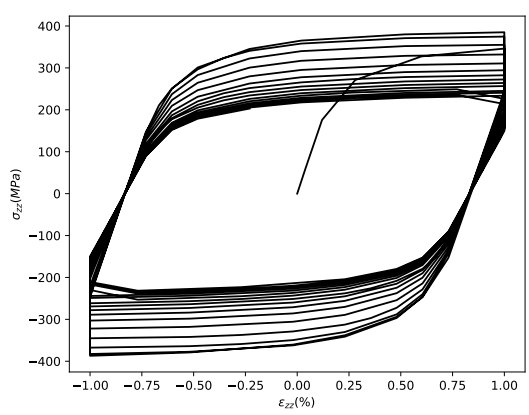

(b)

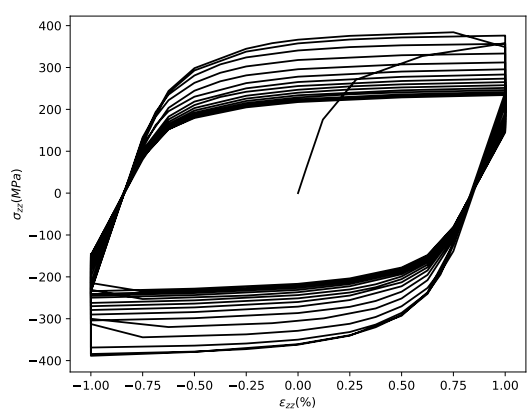

(c)

Figure 4.4: Shown above are the stress-strain curves for the creep-fatigue simulations for models with (a) interfacial damage model only, (b) transgranular damage model only and (c) both interfacial and transgranular damage models.

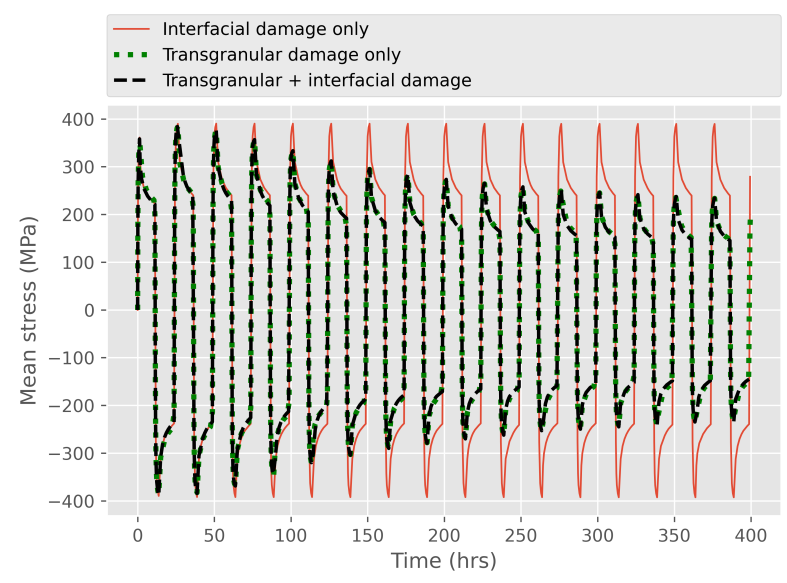

(a)

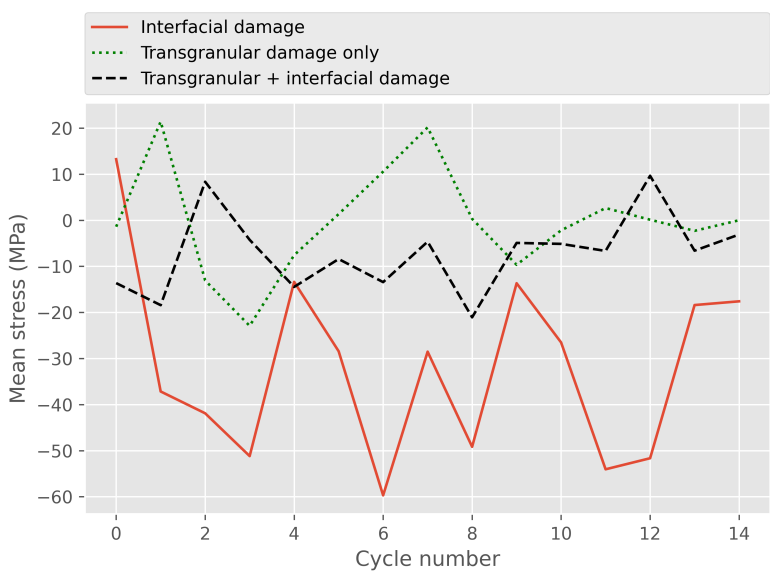

(b)

Figure 4.5: (a) The mean stress in the loading direction is plotted against time for the three damage models considered. (b) The mean stress plotted against the cycle number. 


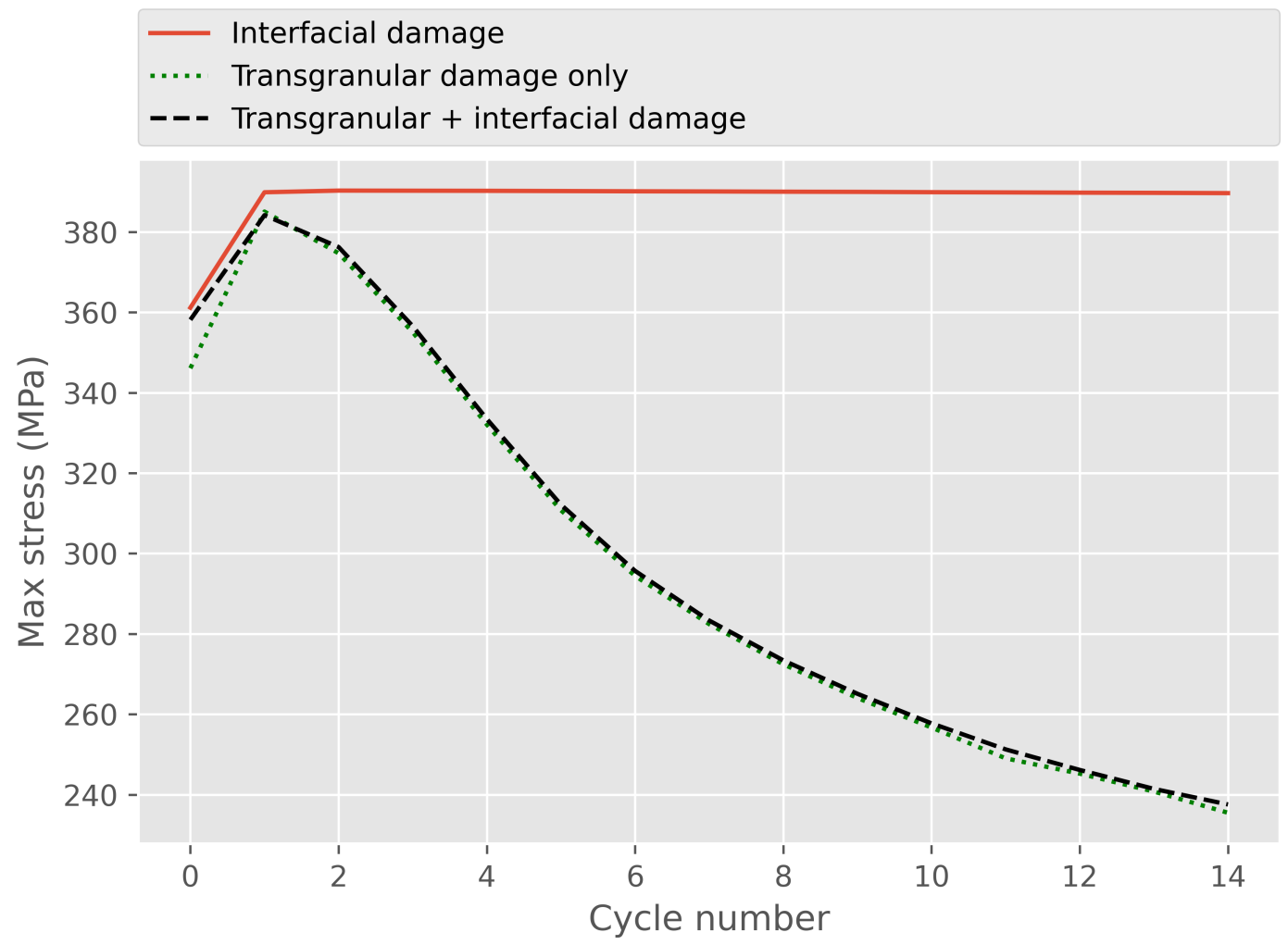

Figure 4.6: The maximum stress in the loading direction is plotted against cycle number for the three damage models considered. 


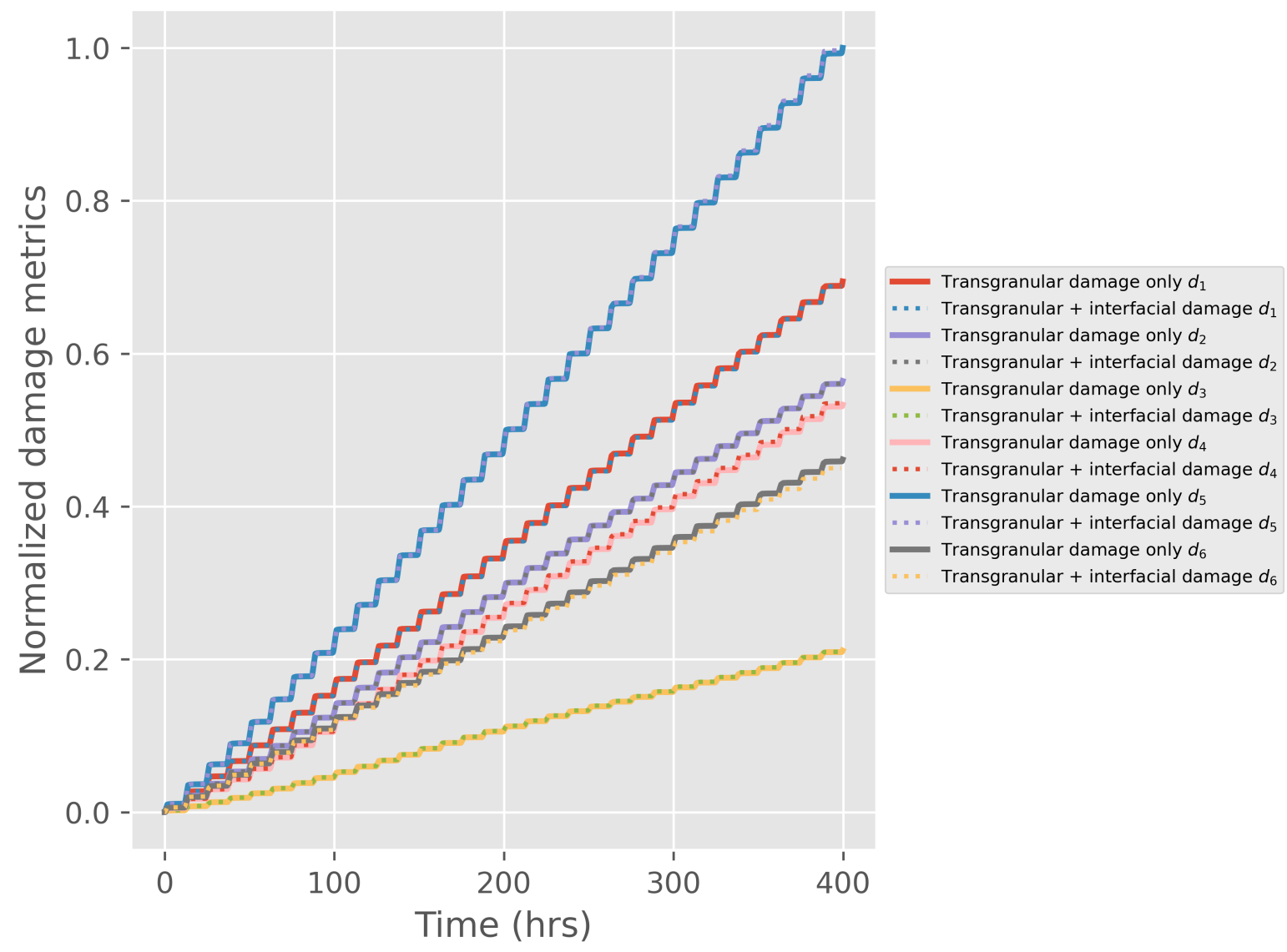

Figure 4.7: Comparison of the transgranular model damage metrics for creep-fatigue simulations.

The damage metrics for the creep-fatigue simulations using the three models are compared in Figure 4.7. The differences in the damage metrics remain relatively small between the two models, suggesting that the influence of creep damage (accommodated in the interfacial model by means of grain boundary cavitation) is small on fatigue damage, particularly in these earlier cycles. The maximum transgranular damage metric is the $d_{5}$ value. The difference between the two models with transgranular damage is more closely observed in Figure 4.8. We observe an initial decrease in this difference followed by a continued increase after about 4 creep-fatigue cycles. The above observation possibly suggests that creep damage dominates towards the earlier cycles and then the fatigue damage takes over. Further, for the purely interfacial damage model, the evolution of the damage metric $D$ is plotted as a function of time and cycle number in Figure 4.9. As expected, there is a continuous increase in the value of $D$ with time and cycles. 


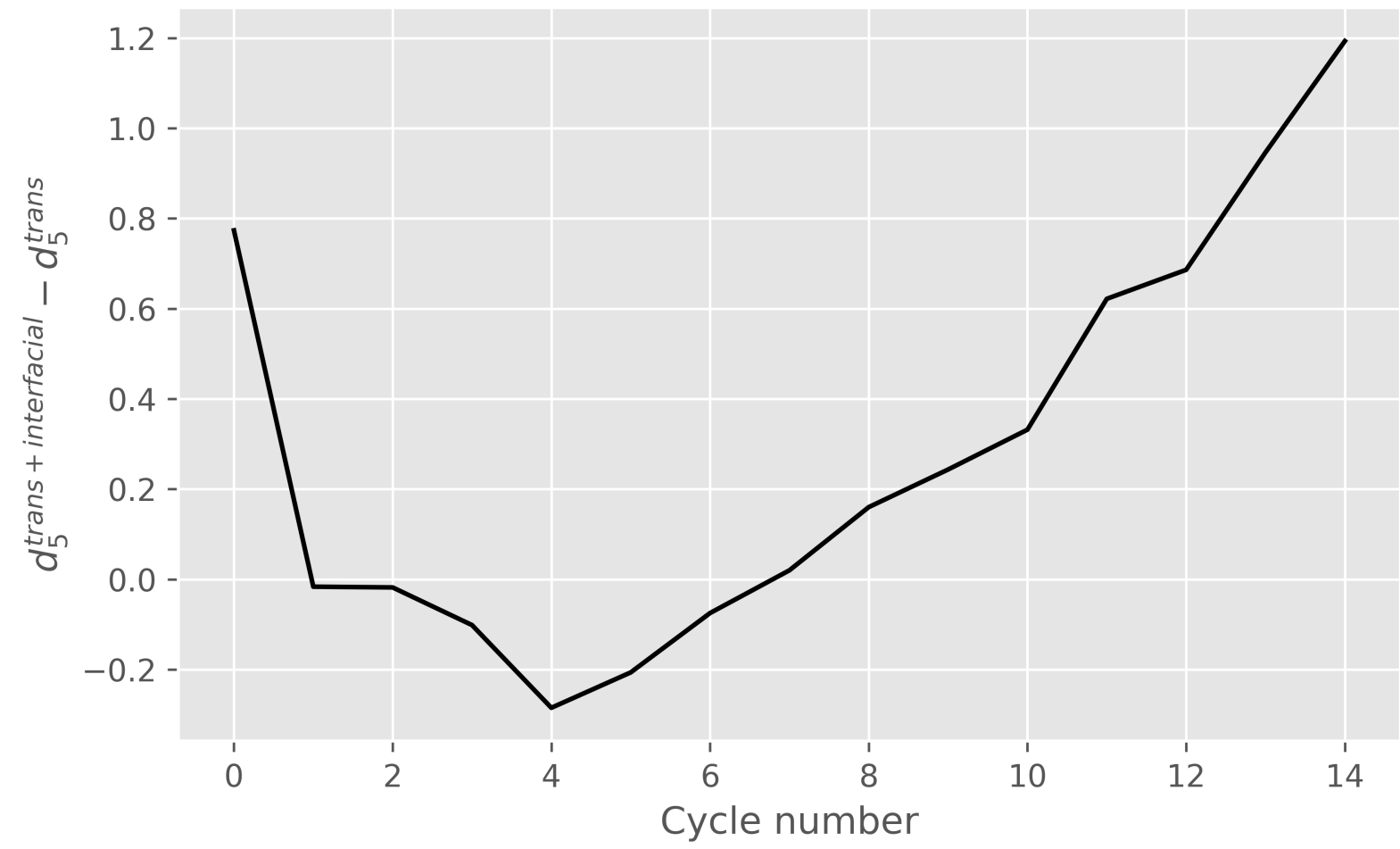

Figure 4.8: Difference between the maximum transgranular damage metrics for the combined transgranular + interfacial model and just the interfacial model.

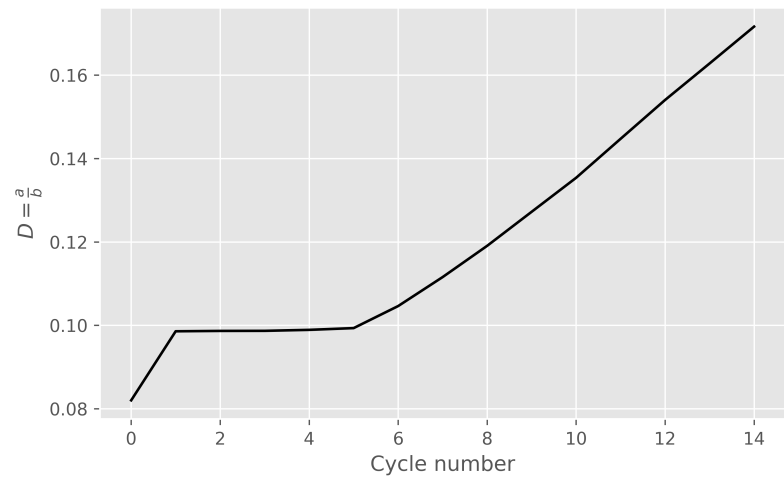

(a)

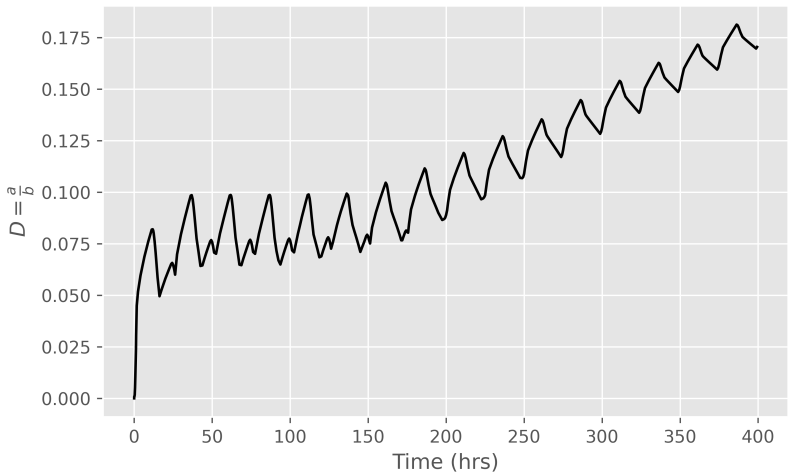

(b)

Figure 4.9: The evolution of the interfacial damage metric as a function of (a) cycle number and (b) time for the model with only interfacial damage model. 
These preliminary results for comparatively few cycles do not reveal a significant creepfatigue interaction likely because the creep cavitation model does not have sufficient time to affect the results. Longer simulations would develop additional creep damage and more significantly affect the cyclic history of the representative element. In addition, these preliminary studies consider only an unrepresentatively small microstructure. Running simulations for larger cells would be necessary to confirm the above observations. 



\section{Conclusions}

This report describes a first-of-its-kind simulation framework for examining creep-fatigue deformation and damage in Grade 91 steel. The framework includes several features developed through past program work:

- Single crystal constitutive models suitable for representing kinematic hardening.

- An interface-cohesive formulation for diffusion-assisted grain boundary cavitation.

as well as a new feature developed in the course of the present work

- A continuum damage mechanics-based formulation for representing transgranular fatigue damage in the CPFEM models.

The report includes several demonstration simulations with the new framework, illustrating the ability of the new model to represent low cyclic fatigue and for the complete modeling framework to represent key processes leading to creep-fatigue interaction.

The simulations presented here are limited in both the size of the represented microstructure and the time/number of cycles considered in the simulation run. The complete model is computationally costly, as it has to maintain and advance a large set of internal variables representing bulk and boundary deformation and damage at every material point in a CPFEM simulation. Running full-scale simulations of low cycle fatigue and short cycle creep-fatigue interaction are feasible given additional time and computational resources. The report identifies polycrystal homogenization as a likely route for simulations covering the transition from low cycle to high cycle fatigue. This route is not easily available for the creep-fatigue simulations including the grain boundary cavitation model as classical homogenization approaches do not explicitly represent the grain boundaries. Another potential solution discussed here are time acceleration/extrapolation strategies, which effective jump through a number of cycles in a single extrapolation step and could be applied to full-scale CPFEM simulations of creep-fatigue interaction. 



\section{Acknowledgements}

The research was sponsored by the U.S. Department of Energy (DOE), under contract No. DEAC02-06CH11357 with Argonne National Laboratory, managed and operated by UChicago Argonne LLC, and under contract No. DE-AC0500OR22725 with Idaho National Laboratory, managed and operated by Battelle Energy Alliance, LLC. Programmatic direction was provided by the Advanced Reactor Technologies Program of the Office of Nuclear Reactor Deployment of the Office of Nuclear Energy (NE).

The authors gratefully acknowledge the support provided by Sue Lesica of DOE-NE, Advanced Materials Lead, Diana Li of DOE-NE, Federal Manager, Microreactor Program,

John H. Jackson of Idaho National Laboratory, National Technical Director, Microreactor Program, and Holly Trellue of Los Alamos National Laboratory, Technology Maturation Technical Area Lead, Microreactor Program.

Helpful discussions with David Parks of MIT are gratefully acknowledged. 



\section{Bibliography}

[1] M. C. Messner and A. Rovinelli. Identify the influence of microstructure on mesoscale creep and fatigue damage. Technical Report ANL-20/49, Argonne National Laboratory, 2020.

[2] A. Rovinelli, M. C. Messner, Ye Guosheng, and T.-L. Sham. Initial study of notch sensitivity of grade 91 using mechanisms motivated crystal plasticity finite element method. Technical Report ANL-ART-171, Argonne National Laboratory, 2019.

[3] T-L Sham and A Needleman. Effects of triaxial stressing on creep cavitation of grain boundaries. Acta Metallurgica, 31(6):919-926, 1983.

[4] Omar Nassif, Timothy J Truster, Ran Ma, Kristine B Cochran, David M Parks, Mark Christian Messner, and TL Sham. Combined crystal plasticity and grain boundary modeling of creep in ferritic-martensitic steels: I. theory and implementation. Modelling and Simulation in Materials Science and Engineering, 27(7):075009, 2019.

[5] Mark Christian Messner, Omar Nassif, Ran Ma, Timothy J. Truster, Kristine Cochran, David Parks, and TL Sham. Combined crystal plasticity and grain boundary modeling of creep in ferritic-martensitic steels: II. the effect of stress and temperature on engineering and microstructural properties. Modelling and Simulation in Materials Science and Engineering, 27(7):075010, 2019.

[6] A. Rovinelli, M. C. Messner, and T.-L. Sham. Initial microstructural model for creepfatigue damage in grade 91 steel. Technical Report ANL-ART-202, Argonne National Laboratory, 2020.

[7] Henry Proudhon, Jia Li, F Wang, A Roos, Vincent Chiaruttini, and Samuel Forest. 3d simulation of short fatigue crack propagation by finite element crystal plasticity and remeshing. International Journal of Fatigue, 82:238-246, 2016.

[8] Jihed Zghal, Hela Gmati, Charles Mareau, and Franck Morel. A crystal plasticity based approach for the modelling of high cycle fatigue damage in metallic materials. International Journal of Damage Mechanics, 25(5):611-628, 2016.

[9] PE McHugh and R Mohrmann. Modelling of creep in a ni base superalloy using a single crystal plasticity model. Computational materials science, 9(1-2):134-140, 1997.

[10] Vikas Hasija, S Ghosh, Michael J Mills, and Deepu S Joseph. Deformation and creep modeling in polycrystalline Ti-6Al alloys. Acta materialia, 51(15):4533-4549, 2003.

[11] Masoud Anahid, Mahendra K Samal, and Somnath Ghosh. Dwell fatigue crack nucleation model based on crystal plasticity finite element simulations of polycrystalline titanium alloys. Journal of the Mechanics and Physics of Solids, 59(10):2157-2176, 2011.

[12] Kai-Shang Li, Run-Zi Wang, Guang-Jian Yuan, Shun-Peng Zhu, Xian-Cheng Zhang, Shan-Tung Tu, and Hideo Miura. A crystal plasticity-based approach for creep-fatigue 
life prediction and damage evaluation in a nickel-based superalloy. International Journal of Fatigue, 143:106031, 2021.

[13] Welding Research Council. Recommended practices in elevated temperature design: a compendium of breeder reactor experiences (1970-1987): Volume II - preliminary design and simplified methods. Technical report, 1991.

[14] American Society of Mechanical Engineers. ASME Boiler and Pressure Vessel Code, Section III, Division 5. 2019.

[15] LJ Carroll, C Cabet, MC Carroll, and RN Wright. The development of microstructural damage during high temperature creep-fatigue of a nickel alloy. International Journal of Fatigue, 47:115-125, 2013.

[16] B Ozmat, AS Argon, and DM Parks. Growth modes of cracks in creeping type 304 stainless steel. Mechanics of Materials, 11(1):1-17, 1991.

[17] Sunggi Baik and R Raj. Mechanisms of creep-fatigue interaction. Metallurgical Transactions A, 13(7):1215-1221, 1982.

[18] P Rodriguez and K Bhanu Sankara Rao. Nucleation and growth of cracks and cavities under creep-fatigue interaction. Progress in materials science, 37(5):403-480, 1993.

[19] Jean-Louis Chaboche. Continuum damage mechanics: Part I — General concepts. Journal of Applied Mechanics, 55(1):59-64, 1988.

[20] Motoki Sakaguchi, Ryota Komamura, Xiaosheng Chen, Mana Higaki, and Hirotsugu Inoue. Crystal plasticity assessment of crystallographic stage i crack propagation in a ni-based single crystal superalloy. International Journal of Fatigue, 123:10-21, 2019.

[21] Shashwat Sinha and Somnath Ghosh. Modeling cyclic ratcheting based fatigue life of hsla steels using crystal plasticity FEM simulations and experiments. International Journal of Fatigue, 28(12):1690-1704, 2006.

[22] Dong-Feng Li, Richard A Barrett, Padraic E O'Donoghue, Noel P O'Dowd, and Sean B Leen. A multi-scale crystal plasticity model for cyclic plasticity and low-cycle fatigue in a precipitate-strengthened steel at elevated temperature. Journal of the Mechanics and Physics of Solids, 101:44-62, 2017.

[23] Florent Bridier, David L McDowell, Patrick Villechaise, and José Mendez. Crystal plasticity modeling of slip activity in Ti-6Al-4V under high cycle fatigue loading. International Journal of Plasticity, 25(6):1066-1082, 2009.

[24] Mohamad Ghodrati, Mehdi Ahmadian, and Reza Mirzaeifar. Three-dimensional study of rolling contact fatigue using crystal plasticity and cohesive zone method. International Journal of Fatigue, 128:105208, 2019.

[25] Jean-Luc Bouvard, Jean Louis Chaboche, F Feyel, and F Gallerneau. A cohesive zone model for fatigue and creep-fatigue crack growth in single crystal superalloys. International Journal of Fatigue, 31(5):868-879, 2009. 
[26] Charles Mareau and Franck Morel. A continuum damage mechanics-based approach for the high cycle fatigue behavior of metallic polycrystals. International Journal of Damage Mechanics, 28(6):838-856, 2019.

[27] Jan G Rots, P Nauta, GMA Kuster, and Johan Blaauwendraad. Smeared crack approach and fracture localization in concrete. HERON, 30 (1), 1985, 1985.

[28] Nailong Zhao, Anish Roy, Weizhe Wang, Liguo Zhao, and Vadim V Silberschmidt. Coupling crystal plasticity and continuum damage mechanics for creep assessment in cr-based power-plant steel. Mechanics of Materials, 130:29-38, 2019.

[29] Lu Feng, Ke-shi Zhang, Guang Zhang, and Hai-dong Yu. Anisotropic damage model under continuum slip crystal plasticity theory for single crystals. International journal of solids and structures, 39(20):5279-5293, 2002.

[30] Ozgur Aslan, NM Cordero, Anais Gaubert, and Samuel Forest. Micromorphic approach to single crystal plasticity and damage. International Journal of Engineering Science, 49(12):1311-1325, 2011.

[31] WAM Brekelmans and JHP De Vree. Reduction of mesh sensitivity in continuum damage mechanics. Acta Mechanica, 110(1):49-56, 1995.

[32] Robert J Asaro. Crystal plasticity. Journal of Applied Mechanics, 50(4b):921-934, 1983.

[33] Andrea Rovinelli, Yoann Guilhem, Henry Proudhon, Ricardo A Lebensohn, Wolfgang Ludwig, and Michael D Sangid. Assessing reliability of fatigue indicator parameters for small crack growth via a probabilistic framework. Modelling and Simulation in Materials Science and Engineering, 25(4):045010, 2017.

[34] Ali Fatemi and Darrell F Socie. A critical plane approach to multiaxial fatigue damage including out-of-phase loading. Fatigue 83 Fracture of Engineering Materials 85 Structures, 11(3):149-165, 1988.

[35] R_A Lebensohn and CN Tomé. A self-consistent viscoplastic model: prediction of rolling textures of anisotropic polycrystals. Materials Science and Engineering: A, 175(1-2): $71-82,1994$.

[36] Deepu S Joseph, Pritam Chakraborty, and Somnath Ghosh. Wavelet transformation based multi-time scaling method for crystal plasticity fe simulations under cyclic loading. Computer methods in applied mechanics and engineering, 199(33-36):2177-2194, 2010. 




\section{Argonne}

\section{Applied Materials Division}

Argonne National Laboratory

9700 South Cass Avenue, Bldg. 212

Argonne, IL 60439

www.anl.gov 